\title{
ORGANIZACIONES INTERPROFESIONALES E INNOVACIÓN AGRÍCOLA: ENTRE LÓGICAS GLOBALES Y TERRITORIALES. EL ROL DE ACSOJA, ASAGIR Y PROARROZ EN ARGENTINA
}

\author{
MARÍA VALENTINA LOCHER ${ }^{1}$ \\ MARTINE GUIBERT ${ }^{2}$ \\ Universidad Nacional del Litoral \\ Université Toulouse - Jean Jaurès
}

\begin{abstract}
Resumo: O artigo analisa o papel das organizações inter profissionais (OIP) nos processos de inovação agrícola. Estes jogadores, relativamente recentes na Argentina, são caracterizados por uma integração vertical dos atores da cadeia agroindustrial e eles têm a particularidade de uma manifesta vocação tecnológica. Mostramos, a partir de uma abordagem comparativa, como esses atores assumem diferentes papéis, dependendo das condições que caracterizam a cadeia que eles representam. O referencial teórico combina abordagens institucionalistas sobre diferentes maneiras de coordenar os processos de inovação com abordagens críticas que permitem dar conta de lógicas globais e territoriais que cruzam os processos de inovação das cadeias. Três casos são analisados: ACSOJA (OIP da cadeia da soja), ASAGIR (OIP da cadeia do girassol) e PROARROZ (OIP da cadeia do arroz). Utilizam-se documentos institucionais de organizações e entrevistas conduzidas pessoalmente com atores como fontes de informação.
\end{abstract}

Palavras-chave: Organizações Inter Profissionais; Inovação Agrícola; Cadeia Agroindustrial; Argentina.

INTERPROFESSIONAL ORGANIZATIONS AND AGRICULTURAL INNOVATION: BETWEEN GLOBAL AND TERRITORIAL LOGICS.

THE ROLE OF ACSOJA, ASAGIR AND PROARROZ IN ARGENTINA.

Abstract: The aim of this paper is to analyze the role of interprofessional organizations (IPO) in the processes of agricultural innovation. IPO shave been recently created in Argentina and they are

${ }^{1}$ Universidad Nacional del Litoral (Argentina).

${ }^{2}$ Université Toulouse - Jean Jaurès (France) - Groupe de recherche Dynamiques Rurales 
characterized by vertically integrating members of an agri-food chain. Also, they have a particular technological vocation. Following a comparative approach we show that these actors take different roles depending on the conditions that characterize the chain. The theoretical framework combines institutionalist approaches on the ways of coordinating innovation processes with critical approaches that allow to show the global and territorial logics that condition the innovation process.

Three cases are analyzed: ACSOJA (IPO of soybeans chain), ASAGIR (IPO of sunflower chain) and PROARROZ (IPO of rice chain).

We use institutional documents and personally conducted interviews with actors as sources of information.

Keywords: Interprofessional Organizations; Agricultural Innovation; Agri-Food Chain; Argentina

\section{Introducción}

Desde la década de 1990, Argentina experimenta un proceso de transformaciones rápidas y profundas de su sector agrícola caracterizadas por importantes incrementos de la productividad, de la producción y de las exportaciones que alcanzan sus niveles históricos más elevados. Este proceso está estrechamente relacionado con la introducción en la agricultura de una serie de innovaciones que han demostrado ser uno de los factores determinantes del crecimiento y del dinamismo del sector. La adopción de estas tecnologías ha implicado asimismo profundas modificaciones en las formas de organización de la producción agrícola, tanto en el seno de las explotaciones como en el sector en general. La característica distintiva de este proceso es la penetración de las formas empresariales en todos los eslabones de las cadenas agrícolas.

Estas transformaciones se corresponden con cambios en el sistema de innovación agrícola, fundamentalmente en lo que respecta a los actores que llevan a cabo los desarrollos tecnológicos ya la manera en que los usuarios acceden a los mismos. En este sentido, los autores que estudian la transformación del sistema de innovación agrícola en Argentina han identificado algunos rasgos predominantes (ANLLÓ et al. 2013; BISANG, 2004; ALEMANY, 2003; THORNTON, 2004; TRIGO, 2005). En primer lugar, desde la década de 1990 se verifica un proceso de "privatización" de los agentes implicados en este proceso, es decir, la participación cada vez mayor del sector privado (especialmente de empresas transnacionales) en el desarrollo y difusión de tecnologías. De manera concomitante ha tenido lugar la reducción de la presencia y del peso del sector público en la investigación agrícola. Por último, se distingue la aparición de nuevos actores y organizaciones (generalmente privadas y sin fines de lucro) dedicados a fomentar y difundir ciertas tecnologías o técnicas particulares.

En este contexto, se observa el surgimiento de organizaciones interprofesionales (OIP) en las cadenas agroindustriales argentinas. Este tipo de estructuras, hasta este momento inexistentes en el país, reúne a gran parte de los actores que participan en 
la producción de un cultivo. Las mismas se forman con el objetivo de abordar problemas e intereses comunes a los agentes de la cadena y poner en práctica estrategias colectivas para resolverlos (RASTOIN y GHERSI, 2010).

Durante el período de grandes transformaciones que ha sido caracterizado más arriba, surgen entre 1994 y 2004, siete organizaciones interprofesionales (OIP): PROARROZ (Fundación de la cadena del arroz, 1994), ASAGIR (Asociación argentina de la cadena del girasol, 2000), IPCVA (Instituto de promoción de la carne vacuna argentina, 2001), COVIAR (Corporación vitivinícola argentina, 2003), ACSOJA (Asociación argentina de la cadena de la soja, 2004), MAIZAR (Asociación argentina de la cadena del maíz, 2004) y ARGENTRIGO (Asociación argentina de la cadena del trigo, 2004).

Creadas bajo diferentes formas institucionales, las OIP pueden estar integradas por productores agrícolas, industriales, proveedores de insumos, universidades y otras instituciones de ciencia y tecnología públicas o privadas, agencias del gobierno de diferentes niveles territoriales o servicios relacionados con la producción. Las OIP representan una novedad en el contexto organizacional argentino ya que, a diferencia de las organizaciones preexistentes (tipo sindicatos), éstas no se basan en las características socioeconómicas relativamente homogéneas de los actores representados (principio de estructuración horizontal) sino en intereses comunes y específicos de una cadena (lógica vertical). Además, en los objetivos expresados en sus estatutos, la vocación innovadora se distingue claramente: las OIP argentinas reconocen a la tecnología como un aspecto central para el desarrollo de las cadenas y, por lo tanto, buscan asegurar su promoción.

El objetivo del presente trabajo es analizar el rol que estas organizaciones juegan en los procesos de innovación de las cadenas, así como los factores que influyen en dichos roles. Se estudian las OIP de tres cadenas distintas: ACSOJA para la de la soja, ASAGIR para la del girasol, PROARROZ para la del arroz.

Las fuentes de información utilizadas son entrevistas a actores realizadas entre 2011 y 2014, documentos institucionales de las organizaciones y el seguimiento regular de la actualidad y de las actividades del sector. La investigación se desarrolló en el marco de una formación doctoral $^{3}$ y de un programa de cooperación franco-argentino ${ }^{4}$.

La presentación se organiza de la siguiente forma: en primer lugar, se repasan brevemente las innovaciones productivas y organizacionales que han transformado el sector agrícola argentino. En segundo lugar, se presentan los lineamientos teóricos que sirven de guía para interpretar los objetos estudiados. En tercer lugar, se analizan las cadenas y el rol del OIP en el proceso de innovación.

\footnotetext{
${ }^{3}$ LOCHER, M. V. (2015). Organisations interprofessionnelles et innovation dans l'agriculture argentine. Les cas de trois filières; soja, riz et tournesol Thèse de doctorat en Études Rurales en Économie. Université Toulouse -Jean Jaurès. 393 p.

4 ProgramaECOS Argentina-Francia $\mathrm{N}^{\circ} \mathrm{A} 11 \mathrm{H} 01$ 2012-2014 «Transformations des systèmes d'innovation agricole et essor des opérateurs privés de recherche et développement".
} 


\section{Innovaciones productivas y gerenciales en el sector agrícola argentino}

De un modo similar a la fase de modernización de los años 50 y 60, luego del estancamiento de las décadas 80 y 90 (BARSKY y GELMAN, 2009), el sector agropecuario argentino conoce desde hace dos decenios, una fuerte suba de sus volúmenes de producción y de la productividad. De unos 40 millones de toneladas producidas en unos 20 millones de hectáreas a inicios de los años 1990, se logró superar la cifra casi mítica de los 100 millones de toneladas en 2010, en unos 35 a 40 millones de hectáreas cultivadas. En algunos cultivos los rendimientos alcanzan niveles internacionales.

El cultivo estrella es la soja que se impuso en la Pampa y en las otras regiones, siendo la producción que legitima la incorporación de tierras hasta ahora no cultivadas o utilizadas para producciones locales (regiones sub-tropicales del Chaco o del Noroeste en particular). El maíz, el trigo, el girasol, la canola no logran competir con la soja en la Pampa agrícola, mientras la ganadería, sea para carne o para leche, es desplazada hacia tierras marginales. Resulta llamativo el avance de la agricultura sobre la ganadería, el abandono frecuente de la rotación pasturas-cultivos, y la búsqueda de valor agregado en la explotación con el desarrollo de actividades complementarias (fábrica de alimentos balanceados feedlots, molinos, biodiesel, etc.) (ANLLÓ et al., 2013).

El paquete tecnológico (siembra directa, semillas transgénicas, herbicida basado en glifosato, etc.) e instrumentos eficientes (financieros, comerciales, etc.) acompañan y hacen posible el avance de la frontera agrícola y la valorización de productos muy demandados por el mercado. El hecho más emblemático es la generalización del arrendamiento de tierras. Se busca aumentar la escala de producción requerida por el modelo tecnológico y por las exigencias de las metas financieras, y se procura el mantenimiento de un nivel adecuado de rentabilidad. El otro hecho destacable es la sub-contratación de las labores agrícolas con la tercerización de la siembra, del seguimiento de los cultivos (pulverización) y de la cosecha. Una encuesta del CEAG de 2012 arroja como resultado que el arrendamiento alcanza al $80 \%$ de los 770 productores encuestados en la región pampeana y la tercerización al $70 \%$ de las tierras trabajadas.

Como se puede suponer, los pequeños productores familiares más vulnerables, tanto pampeanos como de las otras regiones, no entran en el proceso porque no tienen los recursos suficientes, ni los conocimientos necesarios en relación con los nuevos modos de producir. Estos actores, intentan seguir con las actividades tradicionales y regionales o desaparecen. Los censos agrícolas de 1988 y 2002 (INDEC) muestran una pérdida de aproximadamente 50.000 explotaciones en el caso de la región pampeana (134.000 contra 184.000).

Mientras la concentración de la tierra y los modos de producción transforman y cuestionan la figura del agricultor familiar (CLOQUELL et al., 2007; PIÑEIRO y VILLAREAL, 2005; CRAVIOTTI, 2014), la concentración de la producción en 
pocas manos y el atractivo relativo del sector genera una diversificación de los actores, de los que están modificando el manejo de su actividad hasta los que recién han invertido y delegan el acto productivo. Las formas empresariales tienden a caracterizar cada más vez el manejo de las explotaciones, y los actores presentan estrategias diferenciadas en función de objeticos económicos, financieros y patrimoniales (Entre otros, ver REQUIER-DESJARDINS et al., 2014; GUIBERT, 2013; GROSSO et al., 2013; GUIBERT et al., 2011; HERMELO y RECA, 2010; GRAS y HERNÁNDEZ, 2009).

Estos procesos se destacan por su inscripción en un contexto de globalización que acelera los flujos de información y de saberes, que permite acceder a tecnologías de punta y que desemboca en métodos gerenciales inéditos, adaptados a la realidad local y que alteran los modos clásicos de producción. Resulta necesario traer a colación las herramientas teóricas utilizadas para interpretar cómo y porqué las OIP asumen roles diferentes según las condiciones que caracterizan a la cadena que representan.

\section{Cooperación e innovación: entre lógicas territoriales y globales}

La interpretación del rol de las OIP exige que sean movilizados de manera conjunta, tanto enfoques críticos que permiten dar cuenta de los procesos particulares que envuelven el desarrollo de cada cadena agroindustrial, como enfoques institucionalistas que explican las diferentes formas de coordinación de los procesos de innovación.

Ante todo, y considerando que se trata de cadenas productivas globales, debe considerarse que su desarrollo, su sistema de innovación así como las organizaciones que las representan están condicionados por esta realidad global. En otras palabras, se trata de segmentos locales de sistemas productivos de alcance global y, por lo tanto, están constituidas por actores cuyo espacio de acción puede variar, siendo regional, nacional o mundial. En este sentido, los aportes de Harvey (2010) muestran que toda cadena localizada está atravesada por la lógica de acumulación de capital, encarnada en las estrategias de las empresas transnacionales, pero también de las empresas locales.

Harvey (2010) busca producir un análisis geopolítico y crítico del capitalismo. Para él, la globalización es un fenómeno inherente al modo de producción capitalista, en el cual la escala geográfica de acción está en permanente transformación. Este fenómeno implica dos aspectos: el primero, ligado a la propia naturaleza del capitalismo, refiere a la búsqueda permanente de beneficios sin tomar en cuenta consideraciones espaciales, lo que Marx llamó "la aniquilación del espacio por el tiempo". Así, Harvey (2010, p. 125) sostiene que: 
Lo que puede inferirse teóricamente, y que la experiencia histórico-geográfica del capitalismo confirma, es una carrera incesante hacia la reducción, incluso la eliminación de las barreras espaciales, unida a presiones constantes a la aceleración de la rotación del capital.

El segundo aspecto está vinculado a la resolución de las crisis propias al sistema capitalista. Frente a la caída tendencial de la tasa de ganancia, y los consecuentes excesos de capitales que resultan de las crisis de sobreacumulación, la expansión geográfica y la reorganización espacial aparecen come soluciones que pueden aprovecharse para valorizar dichos excesos. Esta lógica de expansión es denominada la lógica del capital.

Estas consideraciones muestran que el proceso de innovación y, por lo tanto, los agentes que lo llevan a cabo, están influenciados por el proceso de reproducción ampliada del capital. Sin embargo, Harvey reconoce también la presencia de instituciones locales que permiten el desarrollo y, al mismo tiempo, condicionan la lógica del capital. En este sentido, admite que el proceso de acumulación requiere una fijación territorial para poder realizar su ganancia:

el capitalismo busca en permanencia crear un paisaje geográfico a fin de facilitar sus actividades en un tiempo dado, solamente para destruirlo y construir un paisaje totalmente diferente en un tiempo ulterior, esto a fin de responder a una sed inextinguible de acumulación del capital que no tiene fin (HARVEY, 2010, p.127).

La idea de fijación da lugar a la noción de lógica político-territorial, y la cual refiere a las instituciones políticas y, a menudo, estatales. La forma en que el capital se desarrolla en un espacio geográfico específico depende de la articulación entre las relaciones propias al modo de producción capitalista (esfera económica) y una esfera extra-económica, fundada en vínculos político-territoriales (BOYER y SAILLARD, 2002; ALLAIRE y MOLLARD, 2006; JESSOP, 2006). Estos vínculos están basados en el reconocimiento mutuo de los actores que pertenecen a una comunidad territorial con límites más o menos definidos, aunque porosos y flexibles. Los vínculos político-territoriales, que pueden constituirse en diferentes niveles territoriales y superponerse, generan reglas e instituciones capaces de organizar diversos aspectos de la producción de riqueza, tales como el proceso de innovación (GILLY y PECQUEUR, 2002). Los procesos socio-económicos que tienen lugar en un territorio determinado se sustentan en una cierta coherencia estructural entre las esferas económica y extra-económica.

Así, la configuración de cada cadena y de su sistema de innovación está condicionada por la interacción de estas dos lógicas: la lógica del capital que 
determina el carácter global de las cadenas, y la lógica político-territorial que caracteriza su anclaje territorial, consolidando diferentes espacios de regulación a nivel nacional, regional o local. Las dos lógicas son complementarias, pero también contradictorias: esta doble condición es esencial para entender la estabilidad y la dinámica del desarrollo de toda cadena y de sus instituciones.

Luego, desde una perspectiva institucionalista, puede afirmarse que la organización de los procesos de innovación, que tienen lugar en el contexto de los sistemas de innovación (SI), se producen combinando distintos mecanismos de integración económica (POLANYI, 1974) que se materializan en diferentes estructuras organizativas. Tres mecanismos organizan las relaciones entre los actores: la jerarquía, el mercado y la cooperación (POWELL, 1990; EBERS, 1997), las cuales se corresponden con tres tipos de actores que participan en los procesos de innovación: el Estado, las firmas y las redes de innovación, respectivamente.

Las redes de innovación aparecen aquí como una forma particular de estructura organizativa de la innovación, caracterizadas por el tipo de vínculo existente entre sus miembros. Las redes se basan en relaciones de cooperación y colaboración sostenidas por el reconocimiento mutuo y los vínculos intersubjetivos entre sus integrantes. En términos generales, el enfoque relacional denomina confianza a dichos vínculos y descubre ciertas condiciones que favorecen su desarrollo, como por ejemplo: la proximidad territorial, la existencia de una historia compartida, la afinidad sectorial, etc. Las OIP pueden ser interpretadas como redes de innovación que actúan en el contexto de los sistemas de innovación de las cadenas productivas agrícolas.

El rol que las OIP tendrán en el proceso de innovación dependerá de la forma en que la lógica del capital y la lógica político-territorial se expresan en los actores y en los mecanismos de coordinación que gobiernan esos procesos de innovación. Teniendo en cuenta estas consideraciones, dos hipótesis han sido formuladas:

*Hipótesis 1: cuando los procesos de innovación (PI) de una cadena determinada, están controlados por firmas guiadas por la lógica del capital, a menudo transnacionales, las OIP tienden a tener un rol poco importante en dichos procesos. En ese caso, las OIP pueden realizar actividades anexas que contribuyan a sostener los PI.

* Hipótesis 2: cuando la OIP tiene un rol preponderante en los PI de la cadena, la lógica político-territorial tendrá una influencia decisiva en la formación y el funcionamiento de la OIP. A la inversa, cuando la OIP no tiene un rol importante, su formación y su funcionamiento tienden a estar subordinados a la lógica del capital que guía los PI.

Para constatar si estas hipótesis resultan adecuadas, es necesario analizar varios casos que sean al mismo tiempo lo suficientemente similares y diferentes para poner en evidencia la influencia de los factores condicionantes de la actividad de las OIP. 


\section{Las cadenas soja, girasol y arroz, SUS OIP y sus sistemas de innovación}

\section{Análisis comparativo entre tres OIP y tres cadenas productivas}

Este trabajo propone un análisis comparativo de tres OIP argentinas: la OIP de la cadena de la soja (ACSOJA), la OIP de la cadena del girasol (ASAGIR) y la OIP de la cadena del arroz (PROARROZ). Estas representan a tres cadenas con contextos geográficos (ver Figura 1) y productivos significativamente distintos: la cadena de la soja nuclea a los actores de la producción del cultivo más difundido (Pampa y otras regiones) y más producido en el país, mostrando un gran dinamismo en los últimos 20 años, principalmente destinado al mercado mundial. La cadena de girasol representa a un cultivo tradicional (Pampa del Suroeste y del Norte) que debe competir con la soja, y ha ido perdiendo posiciones en el ranking de los productos agrícolas argentinos. Por último, la cadena de arroz tiene una ubicación regional bien específica (Mesopotamia), con menor peso en la estructura productiva nacional, pero de gran importancia regional y con fuerte crecimiento en las últimas dos décadas.
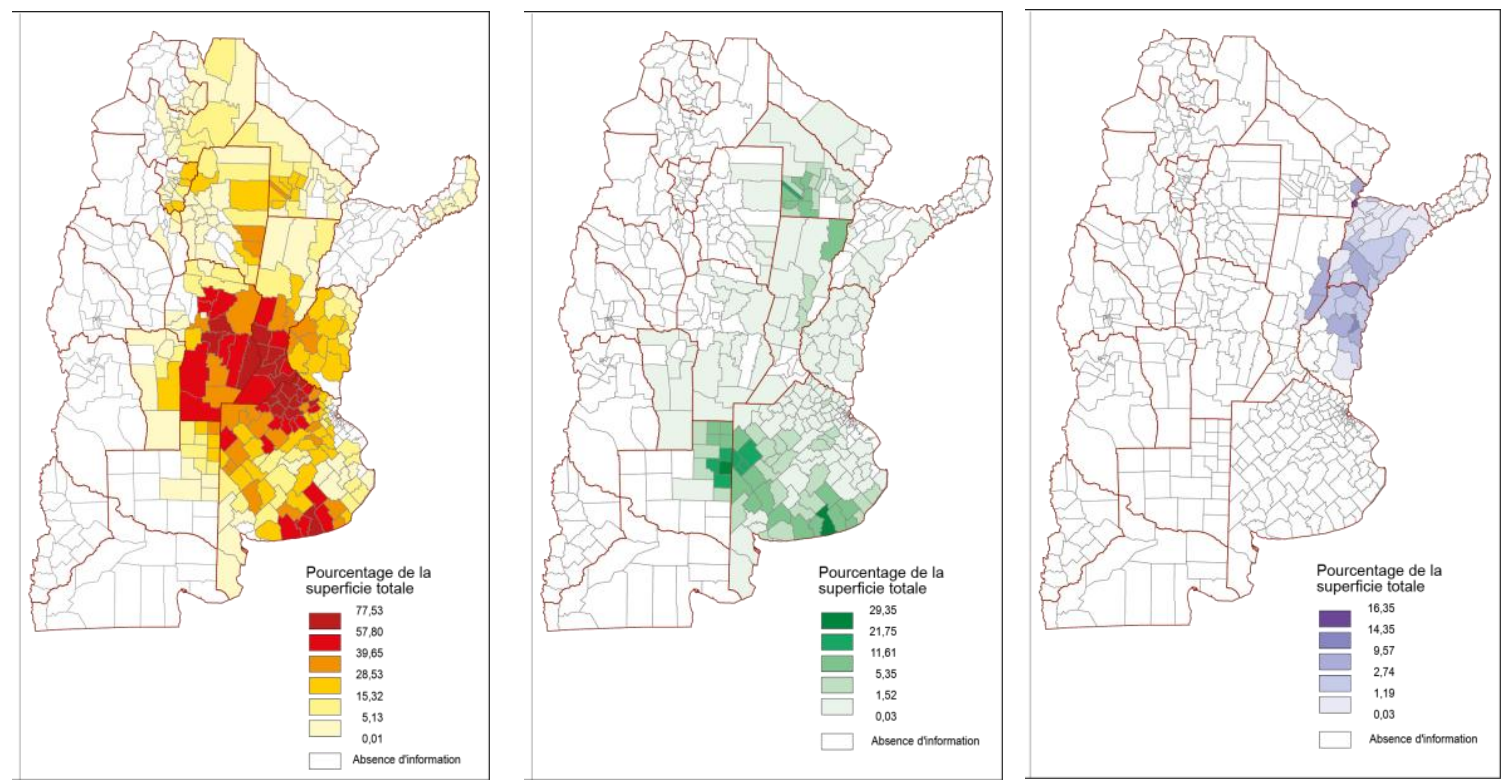

Figura 1: Superficie sembrada de soja (en 2012/13), girasol (en 2010/11) y arroz (en 2010/11), respectivamente, en relación a la superficie de los departamentos.

Fuente: Locher (2015).

Para alcanzar los objetivos definidos, tres niveles requieren ser estudiados: la cadena y su SI, la OIP y los actores que la forman. Distintas fuentes de información se articulan para ello: en primer lugar, datos secundarios de fuentes oficiales y privadas, y trabajos de investigación de referencia, son utilizados para 
precisar las características productivas y tecnológicas de la cadena y su sistema de innovación; en segundo lugar, fuentes documentales: estatutos, convenciones e informes anuales permiten estudiar las OIP y sus actividades. El análisis de las actividades, que resulta clave para observar el papel de las OIP en los procesos de innovación, se realizó a partir de los informes anuales de actividades. Para ello se diseñó una grilla que se utilizó para clasificar cada una de las actividades (Tabla 1) enumeradas en los informes. Se consideró el período de 5 años comprendido entre 2008 y $2012^{5}$.

\begin{tabular}{|c|c|c|}
\hline \multirow{8}{*}{ Tipo de actividad } & \multirow{3}{*}{ Vinculada a la innovación } & Creación \\
\hline & & Difusión \\
\hline & & Financiamiento \\
\hline & \multirow{5}{*}{ No vinculada a la innovación } & Representación de la OIP y comunicación institucional \\
\hline & & Representación corporativa \\
\hline & & Organización interna e integración de la cadena \\
\hline & & Producción y difusión de información \\
\hline & & Desarrollo de la organización \\
\hline \multirow{5}{*}{$\begin{array}{l}\text { Eslabón/es de la } \\
\text { cadena al/los que } \\
\text { está destinada }\end{array}$} & Proveedores de insumos & \\
\hline & Producción primaria & \\
\hline & Industria & \\
\hline & Comercialización & \\
\hline & Toda la cadena & \\
\hline \multirow{3}{*}{ Rol de la OIP } & Organizadora & \\
\hline & Co-organizadora & \\
\hline & Participante & \\
\hline \multirow{3}{*}{$\begin{array}{l}\text { Alcance territorial } \\
\text { de la actividad }\end{array}$} & Regional & \\
\hline & Nacional & \\
\hline & Internacional & \\
\hline
\end{tabular}

Tabla 1: Grilla de análisis de actividades las OIP.

Fuente: elaboración propia.

Por último, respecto a la dimensión de los actores se utilizaron entrevistas en profundidad, cuyo objetivo es principalmente reconocer el significado que los actores otorgan a las actividades de las OIP. Para ello se realizaron 22 entrevistas a

\footnotetext{
${ }^{5}$ El período de análisis fue considerado en función de la disponibilidad de los documentos de las tres organizaciones y, para conservar la homogeneidad, se decidió utilizar sólo los periodos disponibles para los tres casos.
} 
miembros de las cadenas estudiadas y de sus OIP, durante diferentes trabajos de campo llevados a cabo entre marzo de 2011 y octubre de 2013.

\section{La cadena de la soja: dinamismo productivo y tecnológico dominado por la lógica del capital}

Según los datos del SIIA-Minagri ${ }^{6}$, en 2012/13, se produjeron en Argentina casi 50 millones de toneladas de soja, en casi 20 millones de hectáreas, es decir el $60 \%$ de la tierra cultivada. Este cultivo presenta además en promedio un $24 \%$ de aumento de los rendimientos por hectárea si se comparan los de 1994/95 (antes de la autorización comercial de los OGM) y los de 2012/13. Sin embargo, este crecimiento está atenuado por la incorporación de tierras menos fértiles, sobre todo en regiones extra-pampeanas.

El crecimiento en la productividad puede explicarse en buena medida por la incorporación del "paquete tecnológico". Como lo evidencian los datos de Argenbio $^{7}$, por ejemplo, la adopción de semillas genéticamente modificadas alcanzaba en 1999/2000, tres años después de su aprobación en Argentina, el 75 \% de la producción y desde 2002/2003, prácticamente a la totalidad de la soja sembrada en Argentina.

Las semillas, herbicidas y otros productos fitosanitarios fueron introducidos en Argentina por unas pocas grandes empresas transnacionales ${ }^{8}$. Estas firmas desarrollan tecnologías en sus casas matrices y, luego, las adaptan a las condiciones locales para su comercialización o, a través de patentes, licencian sus productos a firmas locales que se ocupan de realizar esta adaptación. Estas firmas se encargan también de la difusión a través de sus centros de servicios, es decir puntos de venta ubicados en las distintas regiones productoras, próximos a los productores y que cumplen además funciones de asesoramiento técnico, pues se encuentran a cargo de ingenieros agrónomos.

Las otras organizaciones que forman parte del sistema de innovación de la cadena contribuyen al mejoramiento y la adaptación de las tecnologías complementarias desarrolladas en el exterior o de proceso. Así, el rol del INTA

\footnotetext{
${ }^{6}$ Los datos están disponibles en el sitio web del Sistema integrado de estadísticas agropecuarias del Ministerio de agricultura, ganadería y pesca: http://www.siia.gov.ar/

${ }^{7}$ Los datos pueden consultarse en el sitio web de ArgenBio: http://www.argenbio.org/

${ }^{8}$ Desde 1995 a 2014, sólo 4 empresas son propietarias de los eventos biotecnológicos de soja que han sido aprobados en Argentina: Nidera S.A., Bayer S.A., Monsanto y BASF S.A (Según datos Comisión Nacional Asesora de Biotecnología Agropecuaria, CONABIA).
} 
(Instituto nacional de tecnología agropecuaria) en esta cadena consiste sobre todo en la mejora en las prácticas de manejo del cultivo (fertilización, tratamiento de insectos y enfermedades, manejo de cosecha). Además, el INTA trabaja en estrecha relación con las empresas semilleras ya que cada nueva variedad debe ser testeada y evaluada en términos de rendimiento y calidad de los granos. El INTA cuenta con recursos humanos y equipamiento para brindar estos servicios a las empresas, quienes obtienen así el aval de una institución que goza de gran prestigio; a cambio ésta recibe ingresos suplementarios ${ }^{9}$.

Otro actor significativo es la Asociación Argentina de Productores en Siembra Directa (AAPRESID), organización sin fines de lucro que ha encabezado el proceso de perfeccionamiento y difusión de la siembra directa. También debe incluirse a la Asociación Argentina de Consorcios Regionales de Experimentación Agrícola (AACREA), conformada por grupos de productores (CREA) que trabajan de forma conjunta para mejorar sus prácticas agrícolas (adaptar el paquete tecnológico a las características de las explotaciones de una región dada).

En síntesis, el sistema de innovación de la cadena de la soja argentina está dominado por las grandes empresas transnacionales que tienen estrategias globales de desarrollo. El rol de los actores nacionales depende de las tecnologías principales controladas por estas empresas. Además, la importancia de la cadena de soja argentina en el contexto internacional puede justificar el interés de las grandes empresas a participar en el país. En efecto, Argentina es el primer exportador mundial de aceite y harina de soja, y el tercer productor mundial, luego de Estados Unidos y Brasil. Por otra parte, el alto número de productores modernizados y aptos para incorporar tecnología ofrecen un gran mercado para recuperar las inversiones hechas en investigación ${ }^{10}$. Tomando en cuenta las nociones introducidas, se trata de un sistema de innovación gobernado por actores guiados por la lógica del capital.

\section{La cadena del girasol: la ineficiencia de la lógica del capital}

El girasol es un cultivo tradicional en Argentina pero ha sufrido en las últimas dos décadas cierto estancamiento debido a un conjunto de factores como las

\footnotetext{
9 “Acá generalmente no se hacen mejoramientos, trabajamos en manejo de cultivos. Trabajamos en control de calidad de semillas. Todo lo que está relacionado a la parte de análisis de calidad hacemos la parte científica y parte servicios, servicio a la comunidad [...] Nos integramos con empresas privadas que nos financian, hacemos convenios de vinculación tecnológica. Las empresas a veces tienen una idea de algo y vienen y nos consultan y nosotros les brindamos el servicio [...] Por ejemplo, hay líneas de investigación que los semilleros traen. $\mathrm{O}$ sea no es que los semilleros resuelven todo solos, sino que el INTA apoya mucho. Por supuesto que ahí si los semilleros ponen plata" (Entrevista realizada a una investigadora de INTA, octubre de 2012).

${ }^{10}$ A modo ilustrativo, puede mencionarse que, según estimaciones del INTA, en 2008 habían 51.031 productores de soja en las principales regiones productoras del país.
} 
tendencias mundiales y nacionales en el consumo, la política comercial fiscal local, la falta de desarrollo de la tecnología y la relativamente débil adopción de innovaciones.

Así, mientras en la década de 1990, la producción se encontraba en torno a los 5 millones de toneladas, desde comienzos del milenio hasta 2012/13, este valor ha sido del orden de los 3,4 millones, ocupando 1,85 millones de hectáreas. En cuanto a los rendimientos por hectárea, prácticamente no han variado desde el año 2000, a lo que se suma una gran variabilidad interanual (datos de SIIA-MINAGRI).

Entre las consecuencias, puede contarse la pérdida de importancia del país como proveedor mundial de sub-productos de girasol: mientras que en 2000, la Argentina proveía el $53 \%$ del aceite comercializado a nivel mundial, en 2012, su participación alcanza sólo el $8 \%$, siendo Ucrania el principal proveedor con el $55 \%$ (BERTELLO, 2012).

El segmento primario de la cadena se vio muy afectado por esta difícil situación. Está integrado en buena medida por productores tradicionales que, a pesar de la tendencia a la concentración horizontal, siguen estando atomizados y deben hacer frente a una industria concentrada con capacidad para fijar los precios. La posibilidad de cambiar o abandonar la actividad, teniendo en cuenta el precio elevado de los arrendamientos, contribuyó a la reducción de la superficie sembrada y dificultó el mantenimiento del número de productores dedicados a este cultivo ${ }^{11}$.

Por su parte, el estancamiento en los rendimientos está relacionado con la situación tecnológica del cultivo. Desde la década de 1990, la oferta tecnológica de semillas e insumos, se concentra en grandes firmas (nacionales y transnacionales) que operan a nivel mundial ${ }^{12}$, pero que, de acuerdo con los actores del sector, dirigen sus inversiones a la investigación aplicada a otros cultivos, lo que explica el relativo retraso tecnológico del cultivo de girasol ${ }^{13}$. De hecho, a diferencia de varios de los principales cultivos, no existen semillas genéticamente modificadas para el girasol, a lo que se suman menores tasas de adopción de otras tecnologías como la siembra directa, que alcanzaba en 2012 al $72 \%$ de la producción según los datos de AAPRESID.

\footnotetext{
${ }^{11}$ Las estimaciones del INTA para 2008 mostraban que había 15.098 productores de girasol en el país.

${ }^{12}$ Los datos del INASE (Instituto Nacional de Semillas) señalan que del total de variedades de semillas de girasol inscriptas desde 2000, $56 \%$ corresponden a firmas de origen extranjero, $38 \%$ a empresas nacionales y $6 \%$ a INTA, la Asociación de Cooperativas Argentinas y la Federación Agraria Argentina.

${ }^{13}$ Las palabras de un productor de girasol de Necochea ilustran esta situación: "El girasol es un cultivo que no acompañó a los otros cultivos en la evolución, por ejemplo no tenemos biotecnología todavía en Argentina (...) No hay biotecnología en girasol porque supongo que los semilleros nos consideran que sea una inversión interesante (...) Entonces fíjate que es lo que le está faltando al girasol. Entró en siembra directa último. La biotecnología no llegó. Y la evolución del cultivo, técnicamente no acompañó a los otros cultivos. Los otros cultivos explotaron y el girasol, no" (Entrevista realizada en diciembre de 2012).
} 
La preponderancia de las firmas en la provisión de tecnologías en el sistema de innovación del girasol, al igual que el de la soja, determina el dominio de la lógica del capital. Un extracto de una entrevista, realizada a un directivo de una de estas empresas en octubre de 2013, ilustra la planificación global de sus estrategias:

(...) la compañía ve a los cultivos con una visión global, y el hecho de que Francia sea un país muy importante en girasol y nuestra empresa esté muy presente y en el resto de Europa, especialmente en el Este y en Rusia, la innovación pasa por los que hacen la investigación para esos mercados. Esas innovaciones son después probadas en la Argentina como un derivado, pero no se hace una investigación básica en Argentina para el girasol argentino. La compañía al ser global, todo programa de investigación y desarrollo está globalizado.

Sin embargo, esta lógica no resuelve satisfactoriamente las necesidades tecnológicas de la cadena, que se encuentra en una situación de relativo retraso.

AACREA y AAPRESID contribuyen con innovaciones relativas al manejo del cultivo. Los actores del sector público (INTA y Universidades) trabajan igualmente en investigaciones sobre enfermedades, insectos y fertilización en girasol. Pero su papel no es suficiente para compensar la falta de inversión privada.

\section{La cadena del arroz: dinamismo productivo y tecnología dominada por la lógica político-territorial}

La situación de la cadena arrocera es muy diferente de las dos anteriores. No ocupa un lugar destacado entre los principales productos agrícolas y las exportaciones del país. Sin embargo, es un cultivo muy importante para la región litoral ${ }^{14}$ del país. Aunque no sin dificultades, y a diferencia de otros productos regionales, el arroz ha logrado aumentar su producción y resistir al avance de otros cultivos extensivos, especialmente la soja.

Así, desde la década de 1990, la producción argentina de arroz ha crecido, no sin oscilaciones, alcanzando actualmente los 1,5 millones de toneladas, mientras que, durante la década de 1980, no se superaban los 400.000 kilogramos. Los rendimientos promedios aumentaron de $5.031 \mathrm{~kg} /$ hectárea en 1994/95 hasta 6.504 $\mathrm{kg} /$ hectárea en 2013/14, lo que equivale a un aumento del $20 \%$ (SIIA-MINAGRI).

\footnotetext{
${ }^{14}$ La región litoral está formada por seis provincias ubicadas en la orilla de los grandes ríos que forman la Cuenca del Plata - ríos Paraná y Uruguay -. Incluye las provincias de Misiones, Corrientes, Entre Ríos (las tres provincias que forman la Mesopotamia argentina, delimitada por los dos ríos mencionados), Chaco, Formosa y Santa Fe.
} 
Las mejoras en la productividad pueden explicarse por el importante proceso de cambio tecnológico que atravesó esta cadena en los últimos 20 años. Las innovaciones introducidas corresponden principalmente a semillas que permiten obtener mayores rendimientos y un producto de mejor calidad, así como en técnicas de manejo de la producción. Sin embargo, a diferencia de los dos casos anteriores, el papel de las empresas en la oferta de tecnologías es secundario. En efecto, el rol del INTA ${ }^{15}$ predomina en el desarrollo de semillas híbridas de arroz. Como veremos con más detalle, estos avances fueron financiados por la PROARROZ, es decir, por la OIP de la cadena del arroz de la provincia de Entre Ríos.

En el sistema de innovación de esta cadena, se destaca la ausencia de inversiones por parte de las empresas, nacionales y transnacionales en investigación. Como ilustra el extracto de entrevista siguiente, los niveles de producción de arroz relativamente bajo respecto a otras regiones del mundo, excluyen a la Argentina de las estrategias globales de estas firmas:

En arroz, nosotros tenemos algunos productos de protección de cultivos, especialmente fungicidas para control de hongos y para tratamiento de semillas. No tenemos semillas, no es un negocio para nuestra empresa en Latinoamérica. En Asia sí, es un player importante y se hace bastante trabajo de investigación. Pero en lo que es Latinoamérica, no es un cultivo principal para la compañía (Entrevista a un directivo de una empresa trasnacional, octubre de 2013).

A pesar de este hecho, la cadena ha logrado resolver sus dificultades tecnológicas mediante la cooperación de actores guiados por la lógica políticoterritorial. El papel del sector privado, especialmente en la oferta de productos de protección de cultivos, es complementaria de las tecnologías generadas mediante esta cooperación.

En definitiva, la descripción precedente muestra las diferencias que existen entre las tres cadenas analizadas, según su característica tecnológica y la lógica que gobierna los procesos de innovación (ver Tabla 2).

\begin{tabular}{|c|c|c|}
\hline & Lógica del capital & Lógica político-territorial \\
\hline Elevado nivel tecnológico & Cadena de la soja & Cadena del arroz \\
\hline Retraso tecnológico & Cadena del girasol & \\
\hline
\end{tabular}

\section{Tabla 2: Cadena, nivel tecnológico y lógica dominante del SI.}

Fuente: Elaboración propia.

${ }^{15}$ Según datos de INTA, $55 \%$ de la siembra de arroz utiliza semillas producidas por INTA, y este valor es muy superior para las provincias de Entre Ríos (86 \%) y de Santa Fe (80 \%). 


\section{Las OIP y sus roles diferenciados en los sistemas de innovación (SI) de cada cadena}

\section{ACSOJA: un actor secundario en el proceso de innovación}

La Asociación argentina de la Cadena de la Soja fue creada por iniciativa de los actores vinculados a la producción de esta oleaginosa, tanto de representantes de instituciones públicas como de empresas privadas. Una característica particular de esta OIP es que nació durante un período de auge de la cadena. De este modo, el objetivo de los socios fundadores fue construir "un mecanismo que agrupe las necesidades de todos aquellos que están vinculados con el negocio de la soja en Argentina" (Entrevista a un dirigente de ACSOJA que participó en su creación, octubre de 2012).

En cuanto a la participación en los procesos de innovación, hemos analizado un total de 109 actividades realizadas por ACSOJA entre 2008 y 2012. La Tabla $\mathrm{n}^{\circ} 3$ sintetiza el resultado de este análisis ${ }^{16}$. De estas actividades, el $22 \%$ están relacionadas con aspectos tecnológicos, mientras que la mayoría se concentra en actividades de representación institucional y política de la cadena. Las actividades se reparten de forma bastante equivalente entre creación de innovaciones (búsqueda de nuevos usos de la soja), difusión de información sobre problemas técnicos puntuales que afecta al cultivo y financiamiento de actividades técnicas realizadas por otras organizaciones, entre las que se puede destacar el banco de germoplasma del INTA.

El principal destinatario de estas actividades es el eslabón primario; sin embargo, el porcentaje de actividades dirigidas a otros actores es relativamente elevado. En general, se trata de acciones realizadas conjuntamente con otros actores, especialmente los de tipo estatal. Entre ellos, el INTA tiene un papel destacado. También se remarca la participación en actividades organizadas por otros actores, lo que se relacione con el perfil de representación que tiene esta OIP.

El alcance mayoritariamente nacional de las actividades muestra que la ACSOJA no tiene una estrategia regional, que tome en cuenta las especificidades de cada zona de producción.

\footnotetext{
${ }^{16}$ Por razones de espacio, sólo se muestran las actividades vinculadas a la innovación ya que son las que sirven estrictamente a nuestro objetivo.
} 


\begin{tabular}{|c|c|c|c|c|}
\hline \multirow{13}{*}{$\begin{array}{l}\text { Actividades } \\
\text { vinculadas a } \\
\text { la innovación }\end{array}$} & \multirow{13}{*}{22,02} & \multirow{3}{*}{ Tipo de intervención } & Creación & 33,33 \\
\hline & & & Difusión & 37,5 \\
\hline & & & Financiamiento & 29,17 \\
\hline & & \multirow{4}{*}{ Eslabón de la cadena } & Proveedores & 16,67 \\
\hline & & & Productores & 37,5 \\
\hline & & & Industria & 25 \\
\hline & & & Toda la cadena & 20,83 \\
\hline & & \multirow{3}{*}{ Alcance territorial } & Regional & 25 \\
\hline & & & Nacional & 66,67 \\
\hline & & & Internacional & 8,33 \\
\hline & & \multirow{3}{*}{ Rol de la OIP } & Organizadora & 25 \\
\hline & & & Co-organizadora & 41,67 \\
\hline & & & Participante & 33,33 \\
\hline
\end{tabular}

Tabla 3: Análisis de las actividades vinculadas a la innovación de ACSOJA entre 2008 y 2012 (en \%).

Fuente: Elaboración propia.

En síntesis, el análisis permite concluir que ACSOJA no posee un proyecto de intervención bien definido en el proceso de innovación. Sus actividades tecnológicas responden a algunos problemas ocasionales y esporádicos, que no privilegian a ningún eslabón específico de la cadena, y no son, en general, iniciativa de la OIP (ver Figura 2).

A pesar de que sus dirigentes la definen como una asociación promotora o facilitadora de la innovación ${ }^{17}$, los actores de la cadena no le reconocen un rol importante en el proceso de innovación. Para los actores industriales (grandes firmas), la asociación no está vinculada a los aspectos tecnológicos sino a aspectos políticos de organización de la cadena. En cambio, los productores y cooperativas consideran que formen parte de esta asociación.

${ }^{17}$ Como lo ilustra este extracto de una entrevista realizada a uno de sus dirigentes: "Yo creo que somos promotores, y desde el hecho que el primer presidente de la asociación es un genetista, me parece que ya está. Creo que tenemos una fuerte impronta en pro de la innovación" (Marzo de 2011). 


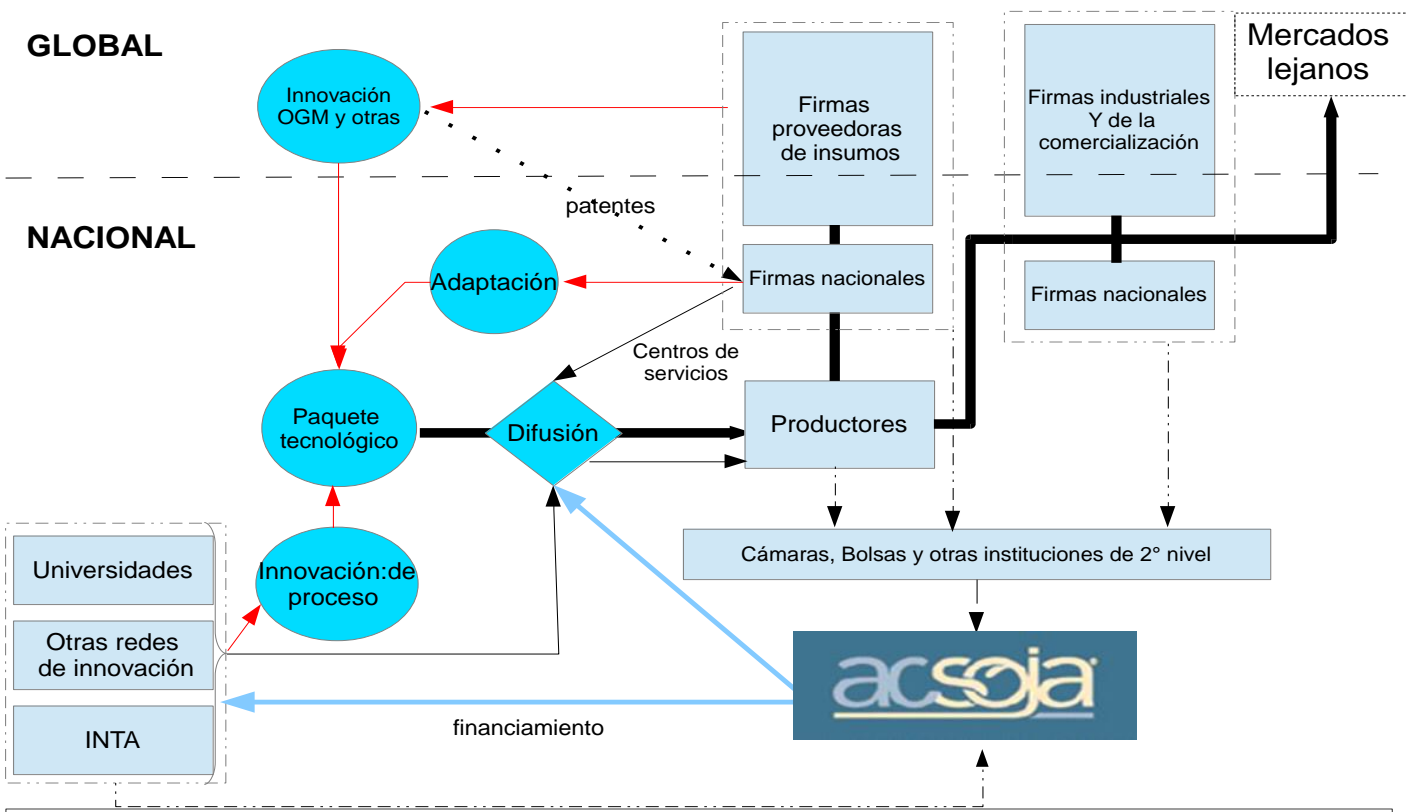

Conocimiento/innovaciones

Difusión

Participación en la OIP

Flujo de producto

Participación de la OIP en los PI

Figura 2: Posición de ACSOJA en el SI y el sistema productivo de la cadena.

Fuente: Locher (2015).

ASAGIR: un actor puente entre actores públicos y actores privados

Movilizados por la difícil situación que el girasol vivía a principios de la década de 2000, algunos actores convocaron a todos los interesados para hacer frente a los problemas. Así, en 2002, se integró Asociación Argentina de Girasol que reúne tanto a organizaciones corporativas como a empresas, especialmente del sector semillero.

En cuanto a las actividades que lleva a cabo ASAGIR, tomando en consideración el mismo quinquenio, se identificaron 124 de las cuales el $21 \%$ están vinculadas a los procesos de innovación de la cadena (ver Tabla 4). Al igual que ACSOJA, esta OIP se dedica mayoritariamente a actividades de representación institucional y política de la cadena. 


\begin{tabular}{|c|c|c|c|c|}
\hline \multirow{13}{*}{$\begin{array}{l}\text { Actividades } \\
\text { vinculadas a } \\
\text { la innovación }\end{array}$} & \multirow{13}{*}{21} & \multirow{3}{*}{ Tipo de intervención } & Creación & 0,00 \\
\hline & & & Difusión & 84 \\
\hline & & & Financiamiento & 16,00 \\
\hline & & \multirow{4}{*}{ Eslabón de la cadena } & Proveedores & 0,00 \\
\hline & & & Productores & 72 \\
\hline & & & Industria & 0,00 \\
\hline & & & Toda la cadena & 28,00 \\
\hline & & \multirow{3}{*}{ Alcance territorial } & Regional & 12 \\
\hline & & & Nacional & 84,00 \\
\hline & & & Internacional & 4,00 \\
\hline & & \multirow{3}{*}{ Rol de la OIP } & Organizadora & 24 \\
\hline & & & Co-organizadora & 40,00 \\
\hline & & & Participante & 32,00 \\
\hline
\end{tabular}

Tabla 4: Análisis de las actividades vinculadas a la innovación de ASAGIR entre 2008 y 2012 (en \%).

Fuente: elaboración propia.

Sin embargo, en lo que respecta a la innovación, ASAGIR tiene un proyecto más concreto de intervención, ya que sus mayores esfuerzos se concentran en el financiamiento y difusión de información hacia los productores sobre las diferentes variedades de semillas disponibles y sus características. Esta actividad se realiza a través de la cooperación que la OIP creó con el INTA y las empresas semilleras, que le ha permitido crear un importante instrumento de decisión para los productores a la hora de elegir la variedad de semilla adecuada para su explotación ${ }^{18}$, la red de evaluación de cultivares.

El público de ASAGIR son los productores agrícolas y sus actividades tienen mayoritariamente alcance nacional. En cuanto a su implicación, se destacan las actividades coorganizadas con otras instituciones (INTA como principal socio).Si bien existe falta de consenso sobre la eficacia de sus acciones, la ASAGIR es reconocida por los miembros de la cadena como un agente implicado en los procesos de innovación ${ }^{19}$.

\footnotetext{
${ }^{18}$ Un dirigente de ASAGIR explica el funcionamiento de este proyecto de la manera siguiente: "Para este trabajo, nosotros tenemos un convenio con el INTA, mediante el cual apoyamos este trabajo. El Inta pone su infraestructura y sus recursos humanos. Y este trabajo también lo hacen las empresas, la diferencia es que esto es público y lo que hacen las empresas es privado, pero nosotros logramos que las empresas bajo un acuerdo de confidencialidad nos den los datos. Entonces logramos reunir 30 mil datos y con eso tenemos una apreciación muy buena del lograble en estas condiciones" (Entrevista, marzo de 2011)

${ }^{19}$ Algunos extractos de entrevistas son ilustrativos: "Y acá acompaño ASAGIR pero para mí no es un motor, no. Para ASAGIR es un muy importante la red. Ellos hacen un buen trabajo de difusión de la red, porque difunden los libritos. Pero se apropiaron de la red. Si, se la conoce como la red ASAGIR. Yo creo que los productores medios y de punta conocen ASAGIR. No sé cuánto les aportara. No sé cuántos leerán los libritos, cuantos miraran internet" (Entrevista a un investigador de INTA, diciembre 2012).
} 
En síntesis, la ASAGIR constituye una forma de intervención que se distingue por su papel en la difusión de información, aunque eso no es suficiente para modificar las condiciones tecnológicas de la cadena de girasol. Sus actividades no pueden modificar la situación de retraso tecnológico en un SI dominado por la lógica del capital pero de manera poco eficaz. La Figura $n^{\circ} 3$ muestra la posición de ASAGIR en el marco de los sistemas de innovación y productivo de la cadena en el espacio de regulación argentino.

\section{GLOBAL}

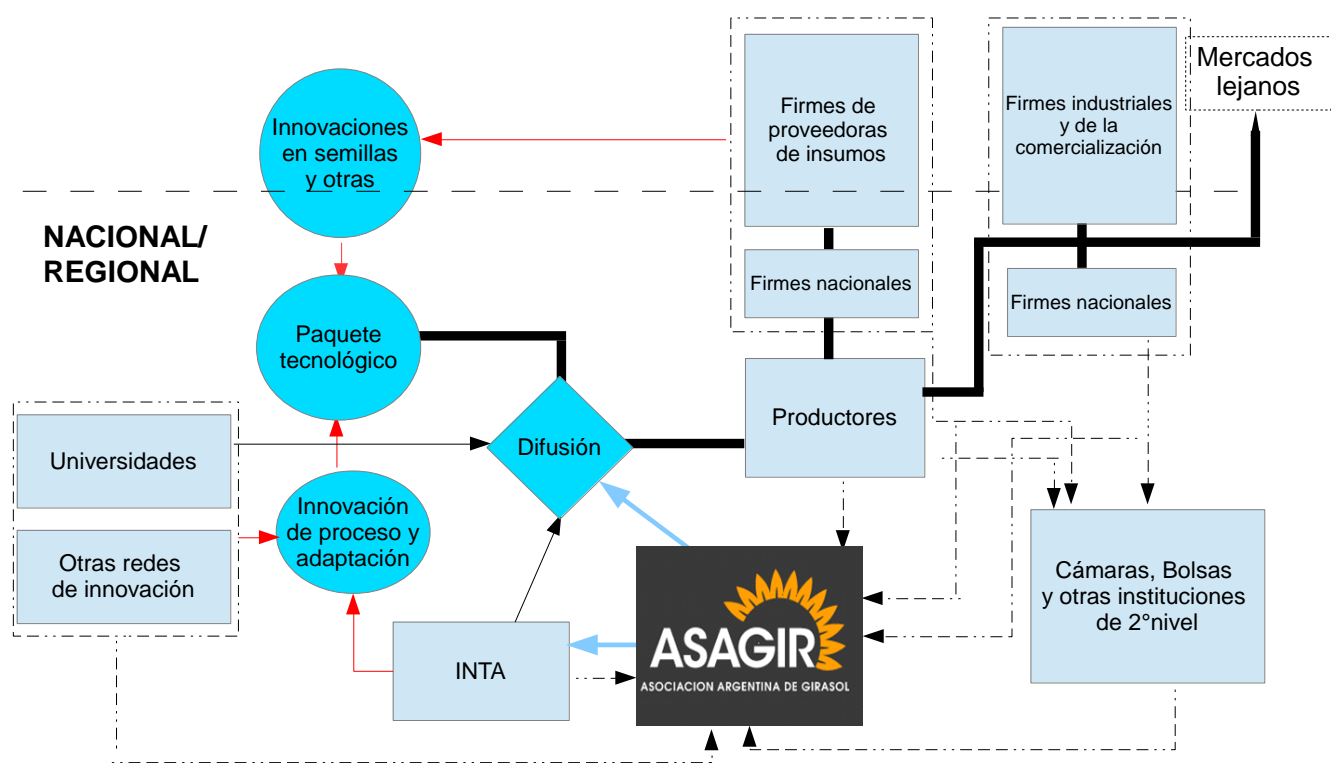

Figura 3: Posición de ASAGIR en el SI y el sistema productivo de la cadena.

Fuente: Locher (2015).

\section{PROARROZ: el actor central de una verdadera red de innovación}

La PROARROZ fue creada como respuesta del sector productivo a la crítica situación que sufrió durante la década de 1990. Los productores y las empresas

\footnotetext{
“ASAGIR está apuntando a algunos proyectos de investigación, pero la mira la deben tener mal, porque están apuntando hace mucho tiempo y no se concreta" (Entrevista a un productor de girasol, diciembre 2012).

"ASAGIR es importante dentro del productor de girasol. El tipo que hace girasol tiene muy presente el tema de lo que es ASAGIR y la información de la red de ASAGIR. Pero el tipo que no hace girasol, capaz que no le llega el mensaje de ASAGIR. Creo que el poder de marketing que tienen estas cadenas no es muy fuerte fuera de la cadena. No van a convencer a alguien de hacer girasol" (Entrevista a un proveedor de insumos de Necochea, diciembre 2012).
} 
industriales, principalmente de la provincia de Entre Ríos, comenzaron a reunirse y buscar soluciones, identificando al atraso tecnológico del sector como una de las dificultades más graves. Los esfuerzos fueron dirigidos a este problema y PROARROZ logró involucrar a los actores públicos y privados de la cadena. Sin embargo, esta fundación se limita a la provincia de Entre Ríos, ya que sólo el gobierno de esta provincia decidió promulgar una ley, la número $\mathrm{n}^{\circ} 9228$, que regula la contribución obligatoria de todo el sector productivo al financiamiento de las actividades de PROARROZ. La OIP representa al sector de la provincia, aunque las tecnologías desarrolladas se difunden al resto de las provincias productoras, incluso en otros países.

El análisis de 123 actividades para el mismo período que en los otros dos casos, arroja un resultado diferente: el $61 \%$ están dirigidas al proceso de innovación.

Estas actividades se concentran en el financiamiento y la difusión. Trabajando en estrecha colaboración con el INTA, se produjeron las variedades de semillas mejoradas que se utilizan en la Argentina, y también se exportan. Estas exportaciones resultan una fuente de ingreso clave que permite mantener el financiamiento del sistema ya que, a través de un convenio con la firma BASF, ésta se encarga de la venta de semillas en el resto del mundo, pagando regalías al INTA que es el propietario de la patente.

PROARROZ también ha invertido en otras actividades relacionadas con el proceso de la innovación. Por ejemplo, ha contratado a un equipo de ingenieros agrónomos que asesoran técnicamente a los productores, jugando un papel clave en la difusión. En este sentido, la OIP actúa como nexo, acercando las necesidades de los productores (principales destinatarios de sus actividades) al sector de ciencia y tecnología. Cabe destacar que la OIP ha estado a cargo de la organización del $84 \%$ de estas actividades.

En síntesis, el perfil de PROARROZ se diferencia del de las otras dos organizaciones por su significativa influencia en los procesos de innovación. Esta OIP se ubica al frente de dicho proceso, participando en sus distintas etapas. Este rol es reconocido por los actores de la cadena, quienes coinciden en asignar un papel clave a la OIP en la puesta en marcha y el desarrollo del proceso de innovación ${ }^{20}$. La centralidad de esta OIP en la organización de la red de innovación es ilustrada en el Figura 4.

\footnotetext{
${ }^{20}$ Algunos extractos de entrevistas permiten ilustrar esta situación: "La fundación nos proveía en algún momento de recursos, también eran las demandas que provenían de la fundación que en definitiva terminan siendo la representación de distintas áreas de la producción o de la industria, respecto de lo que había que lograr. Yo creo que en esto ha sido una cuestión complementaria en el sentido que, bueno, la fundación aportando ya sea recursos o capacidad de difusión, y por otro lado estableciendo demandas concretas" (Entrevista a un investigador de INTA, octubre de 2012).

"Y se maneja muy bien, es una institución muy transparente, muy cristalina. Justamente al haber muchos involucrados por ahí es más fácil de controlar, es más fácil que los fondos se manejen bien. Si no fuera por PROARROZ recién hubiéramos estado arrancando en el 2002 o 2003 con la genética,
} 


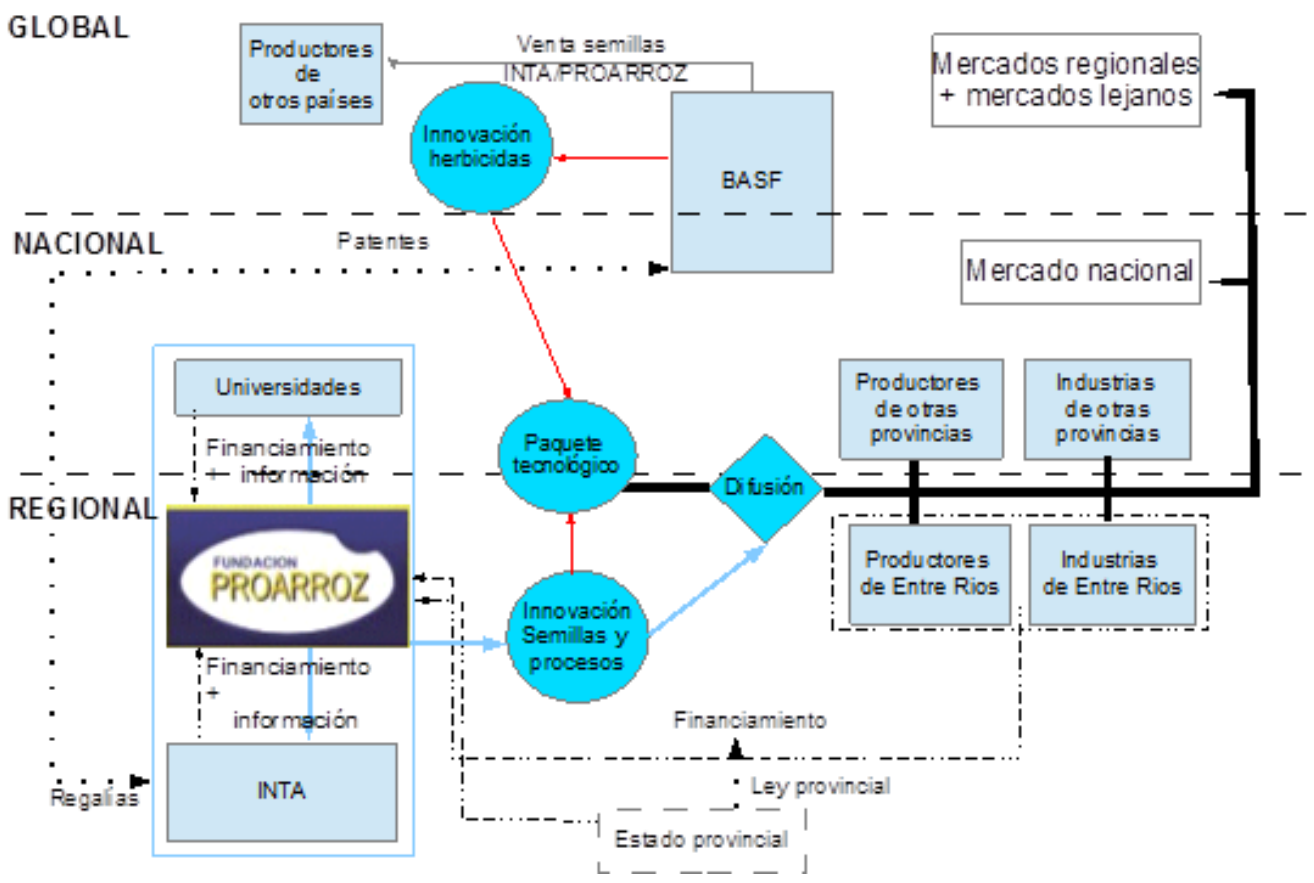

Figura 4: Posición de PROARROZ en el SI y el sistema productivo de la cadena.

Fuente: Locher (2015).

\section{Conclusión}

Para poder ofrecer una interpretación al rol de las OIP en la obtención y difusión de innovaciones, hemos identificado a las lógicas que pilotan los procesos.

En los casos de ACSOJA y ASAGIR, las actividades tecnológicas tienen un peso reducido. Estas OIP pueden contribuir a los procesos de innovación con algunas actividades de apoyo, pero no son fundamentales para su funcionamiento. Se puede decir que son OIP no tecnológicas. En cambio, la participación de PROARROZ en casi todas las etapas del proceso de innovación, a través de la

estaríamos atrasados. Ese mantenimiento que se hizo, a través de esos recursos se lograron por lo menos dos variedades: cambá, puitá y el gurí que es más nuevo" (Entrevista a un productor de arroz, octubre 2012).

"El PROARROZ es uno de los principales generadores de las mejoras en el sector arrocero; tiene tres variedades nuevas que eso ha ayudado primero en la producción primaria, y segundo en la industrialización. Hoy tenemos materia prima como te decía al principio, muy superior a la de los años 90, gracias estas investigaciones del PROARROZ" (Entrevista al gerente de un molino, octubre de 2012). 
financiación de las actividades de investigación del INTA y de las Universidades, la multiplicación de semillas y la difusión de las mejores técnicas a los productores de arroz, determina su perfil claramente tecnológico.

Llegamos al resultado según el cual, cuando la lógica del capital controla el proceso de innovación, dados la presencia y los intereses globalizados de las empresas transnacionales que dominan la cadena, el papel de la OIP es reducido.

Se observó que el caso de la cadena de la soja es un ejemplo donde el pleno despliegue de la lógica del capital resuelve los problemas tecnológicos de la cadena, permitiendo su desarrollo. La ACSOJA tiene un papel secundario, complementario al proceso de innovación dominado por las empresas transnacionales.

En el caso de la cadena del girasol, si bien la lógica del capital es dominante en el proceso de innovación, ésta no logra resolver los problemas tecnológicos que afectan al desarrollo del sector. El rol de ASAGIR también es secundario y no es suficiente para superar estas dificultades. En consecuencia, la lógica del capital sólo permite comprender parcialmente el papel de ASAGIR. Es necesario entonces identificar una dimensión interna a la OIP que permita responder a esta pregunta.

El caso de la cadena del arroz nos ayuda a completar el análisis. En primer lugar, se observó que la lógica del capital está ausente. Sin embargo, esta cadena ha experimentado un acelerado proceso de cambio tecnológico. En segundo lugar, la tecnología de esta cadena fue desarrollada por las instituciones públicas articuladas y financiadas por PROARROZ. Así, la OIP tiene un papel muy importante en el proceso de innovación.

Al mismo tiempo, para el caso de PROARROZ, la presencia, entre los fundadores, de empresas locales, de cooperativas y del gobierno provincial, muestra la importancia de las relaciones basadas en pertenencia territorial que sostienen la cooperación institucionalizada en la OIP. La institucionalización, a través de la legislación provincial, y la financiación, por parte del sector productivo de Entre Ríos, en su conjunto, refuerzan esta idea. La fuerza de estos vínculos es una condición necesaria para que las organizaciones basadas en la lógica políticoterritorial puedan tener un papel importante en los sistemas de innovación en los cuales la lógica del capital no proporciona las tecnologías necesarias.

En contraste, en el caso de ASAGIR, el análisis no revela la presencia de vínculos fundados en los atributos territoriales. El espacio de acción de los actores que conforman esta OIP es nacional o global. La falta de esos vínculos contribuye a comprender el papel de ASAGIR en el proceso de innovación. 


\section{Bibliografía}

ALEMANY, C. (2003) Apuntes para la construcción de los períodos históricos de la Extensión Rural del INTA. In: THORNTON, R.; CIMADEVILLA, G. (eds.). $L a$ Extensión Rural en Debate. Concepciones, retrospectivas, cambios y estrategias para el MERCOSUR. Buenos Aires: Ediciones INTA.

ANLLÓ, G. (2013) Cambio de paradigma tecno-productivo y ¿crisis de representación? Nuevas y viejas entidades de representación de la actividad agrícola. In: ANLLÓ, G.; BISANG, R.; CAMPI, M. (coords.). Claves para repensar al agro argentino. Buenos Aires: EUDEBA. pp. 259-283.

ANLLÓ, G.; BISANG, R.; CAMPI, M. (coords.). (2013) Claves para repensar el agro argentino. Buenos Aires: EUDEBA.

BARSKI, O.; GELMAN, J. (2009) Historia del agro argentino. Desde la Conquista hasta fines del siglo XX. Buenos Aires: Suramericana.

BERTELLO, F. (2012) Bajan las exportaciones de aceite de girasol y advierten que podrían cerrar plantas. La Nación: economía. [En línea] 28 mai. http://www.lanacion.com.ar/1695143-bajan-las-exportaciones-de-aceite-de-girasoly-advierten-que-podrian-cerrar-plantas

BISANG, R. (2004) Innovación y estructura productiva: la aplicación de biotecnología en la producción agrícola pampeana argentina. In: BÁRCENA, A.; KATZ, J.; MORALES, C.; SCHAPER, M. (eds.) Los transgénicos en América Latina y el Caribe: un debate abierto. Santiago de Chile: CEPAL. pp. 71-106.

BISANG, R.; ANLLÓ G.; CAMPI, M. (2010) Organización del agro. La transición de un modelo de integración vertical a las redes de producción agrícola. In: RECA, L. G.; LEMA, D.; FOOD, C. (eds.) El crecimiento de la agricultura argentina Medio siglo de logros y desafios. Buenos Aires: FAUBA. pp. 231-254.

CEAG/Univ. Austral. (2012) Encuesta sobre las necesidades del productor agropecuario argentino. Disponible en: http://www.austral.edu.ar/cienciasempresariales-ceag/proyecto-de-investigacionsobre-las-necesidades-del-productor-agropecuario-argentino-2-edicion/

CLOQUELL, S.; ALBANESI R.; PROPORSI, P.; PREDA, G.; DE NICOLA, M. (2007) Familias rurales. El fin de una historia en el inicio de una nueva agricultura. Rosario: Homo Sapiens.

CRAVIOTTI, C. (comp.). (2014) Agricultura familiar en Latinoamérica. Continuidades, transformaciones y controversisas. Buenos Aires: Ciccus.

EBERS, M. (1997) Explaining Inter-Organizational Network Formation. In: (ed.) The Formation of Inter-Organizational Networks. Oxford: Oxford University Press. pp. 3-40,

GILLY, J.-P.; PECQUEUR, B. (2002) La dimension locale de la régulation. In: BOYER, R.; SAILLARD, Y. (eds.) Théorie de la régulation: l'état des savoirs. Paris: La Découverte. pp. 304-312. 
GRAS, C.; HERNÁNDEZ, V. (coords.). (2009) La Argentina rural - de la agriculturafamiliar a los agronegocios. Buenos Aires: Ed. BiblosSociedad.

GROSSO, S.; BIASIZO, R.; LOCHER, V. M.; GUIBERT; M.; BESSON, M. E.; LAUXMANN, S. (2013) Cambios productivos y organizacionales en los actores del sector agropecuario pampeano. In: ARRILlAGA, H.; CASTAGNA, A.; DELFINO, A.; TROCELLO, G. (coords.). La nueva agricultura y la reterritorialización pampeana emergente. Santa Fé: Ed. UNL. pp. 65-103.

GUIBERT, M. (2013) Les formes associatives de production agricole en Argentine et en Uruguay: entre territoire et réseau ? Études rurales, n ${ }^{\circ} 191$. pp.77-90. Traduction en anglais: Farmers'Networks in Argentina and Uruguay: at the crossroads of territories and networks. http://cairn-int.info/abstractE_ETRU_191_0077--farmers-s-networks-in-argentina.htm

GUIBERT, M.; SILI, M.; ARBELETCHE, P.; PIÑEIRO, D. y GROSSO, S. (2011) Les nouvelles formes d'agricultures entrepreneuriales en Argentine et en Uruguay, Économies et Sociétés, n³3. pp.1813-1831.

HARVEY, D. (2010) Le nouvel impérialisme. Paris: Prairies ordinaires. (Penser/Croiser)

HERMELO, D.; RECA, L, (2010) Asociaciones productivas (APs) en la agricultura: una respuesta dinámica a fallas de mercado y al cambio tecnológico », In: RECA, L. G.; LEMA D. Y FOOD, C. (eds.) El crecimiento de la agricultura argentina. Medio siglo de logros y desafíos, Buenos Aires: FAUBA. pp. 207-229.

JESSOP, B. (2006) Spatial fixes, temporal fixes and spatio-temporal fixes. Department of Sociology, Lancaster University. Disponible en: <http://www.comp.lancs.ac.uk/sociology/papers/jessop-spatio-temporal-fixes.pdf> Consultado el: 13/04/2013.

LOCHER, M. V. (2015) Organisations interprofessionnelles et innovation dans l'agriculture argentine. Les cas de trois filières; soja, riz et tournesol. Thèse de doctorat en Études Rurales en Économie. Université Toulouse - Jean Jaurès. 393 p. MUZLERA, J. y SALOMÓN, A. (2014) Sujetos sociales del agro argentino. Configuraciones históricas y procesos de cambio. Rosario: Prohistoria

MUZLERA, J. (2013) La modernidad tardía en el agro pampeano: sujetos agrarios y estructura productiva. Bernal: UNQ.

PIÑEIRO, M.; VILLAREAL F. (2005) Modernización agrícola y nuevos actores sociales, Ciencia Hoy, no 87. pp. 32-36.

POLANYI, K. (1974) El Sistema económico como proceso institucionalizado. In: GODELIER, M. Antropología y economía. Barcelona: Anagrama. pp. 155-178.

POWELL, W. (1990) Neither market nor hierarchy Network forms of organization. Research in Organizational Behavior, vol. 12. pp. 295-336.

RASTOIN, J-L.; GHERSI, G. (2010) Le système alimentaire mondial. Paris: Quae.

REQUIER-DESJARDINS, D.; GUIBERT, M. et BÜHLER, E. A. (2014) La diversité des formes d'agricultures d'entreprise au prisme des réalités sud- 
américaines. Économie rurale, $\mathrm{n}^{\circ}$ 344. pp. 45-60. http://economierurale.revues.org/4506

THORNTON, R. (2004) INTA en busca de sus vínculos. De públicos a agentes de desarrollo. In: CIMADEVILLA, G. Y CARNIGLIA, E. (eds.). Comunicación, Ruralidad y Desarrollo. Buenos Aires: Ediciones INTA. pp. 307-329.

TRIGO, E. (2005) Consecuencias económicas de la transformación agrícola, Ciencia Hoy, $\mathrm{n}^{\circ} 87$. pp. 46-51. 